\title{
REAL-TIME SPECIFICATIONS OF THE GEOSPACE ENVIRONMENT
}

\author{
Y. KAMIDE ${ }^{1}$, E. A. KIHN ${ }^{2}$, A. J. RIDLEY ${ }^{3}$, E. W. CLIVER ${ }^{4}$ and Y. KADOWAKI ${ }^{1}$ \\ ${ }^{1}$ Solar-Terrestrial Environment Laboratory, Nagoya University, Toyokawa 442-8507, Japan \\ ${ }^{2}$ NOAA National Geophysical Data Center, Boulder, Colorado 80305-3328, U.S.A. \\ ${ }^{3}$ Space Physics Research Laboratory, The University of Michigan, Ann Arbor Michigan \\ 48109-2143, U.S.A. \\ ${ }^{4}$ AFRL/Space Vehicles Directorate, Hanscom AFB, Massachusetts 01731-3010, U.S.A.
}

\begin{abstract}
We report the recent progress in our joint program of real-time mapping of ionospheric electric fields and currents and field-aligned currents through the Geospace Environment Data Analysis System (GEDAS) at the Solar-Terrestrial Environment Laboratory and similar computer systems in the world. Data from individual ground magnetometers as well as from the solar wind are collected by these systems and are used as input for the KRM and AMIE magnetogram-inversion algorithms, which calculate the two-dimensional distribution of the ionospheric parameters. One of the goals of this program is to specify the solar-terrestrial environment in terms of ionospheric processes, providing the scientific community with more than what geomagnetic activity indices and statistical models provide.
\end{abstract}

Key words: geomagnetic storm, ionosphere, magnetosphere, solar wind, space weather, substorm

\section{Introduction}

The integrated understanding of complex interactions that couple the solar wind and the earth's magnetosphere and ionosphere is essential for space weather predictions. It is often said, however, that space weather research is behind surface (or troposphere) weather research by some fifty years in terms of their ability of quantitatively forecasting, for example, tomorrow's weather. To monitor the degree of how much the geospace is disturbed, the scientific community is still relying heavily on geomagnetic indices, such as $K p, D s t$, and $A E$, which are scalar quantities expressing only global magnetic activity, although we are all aware that magnetospheric and ionospheric disturbances are highly localized.

Space weather has recently been becoming increasingly important in many respects, including the needs from the modern high-technology society that depends on satellite communications. This indicates that even though we are still far from a complete understanding of energy flow/transformation processes in the entire solarterrestrial system, we are being expected by society to make predictions, at least, of major geomagnetic storms. It is thus very important to realize that the terminology 'space weather studies' be used to mean both basic research of solar-terrestrial relationships and its applications for space weather predictions including the ad-

Space Science Reviews 107: 307-316, 2003.

(C) 2003 Kluwer Academic Publishers. Printed in the Netherlands. 
vancement of numerical modeling techniques and the construction of algorithms for predicting space 'events'.

At the Solar-Terrestrial Environment Laboratory (STEL), space weather studies are being conducted in basically two ways: understanding basic multi-scale processes occurring over the boundaries of various plasma regions in the solarterrestrial system, and developing algorithms to predict geomagnetic storms/substorms. To promote these projects, STEL has installed a high-technology computer system, called Geospace Environment Data Analysis System (GEDAS). It represents a way to promote integrated studies by combining ground-based and satellite-based observations as well as modeling and simulation research. GEDAS intends to connect similar systems throughout the world on a near real-time basis. It is important to note that what is needed in space weather predictions is not only specifying global disturbances but also locating a particular phenomenon observed at one site within a global perspective.

The purpose of this paper is to report on-going research projects, in particular, of ionospheric electric fields and currents, using the GEDAS in collaboration with other institutions around the world.

\section{What is GEDAS?}

While observations from the earth's surface are considered to be a type of 'remote' sensing for solar-terrestrial processes and thus indirect, they nevertheless generate high spatial/temporal resolutions. On the other hand, satellite observations, being in-situ and 'direct,' provide only 'point' measurements along satellite orbits. It is thus necessary to evaluate crucially merits/demerits of each observation. What GEDAS does in practice can be summarized in the following way:

1. Local and global viewpoints: Without identifying the location of what a particular satellite is measuring within the entire solar-terrestrial system, one may not be able to discuss the physics of these measurements self-consistently. It is expected that by relying on GEDAS in which all available data are compared, one is able to pinpoint the location of localized, explosive phenomena, such as substorm expansions, within large-scale energy flows and transformation.

2. Tests for real-time data in simulation models: To understand nonlinear interactions among various plasma regions in the system, numerical modeling using basic equations is required. Under the GEDAS system, real-time data can be used as initial and boundary conditions for computer simulations, permitting researchers to forecast space weather events that may take place in the near future. Any researcher can be a project leader, using the real-time resources available through GEDAS to drive models or applications in their own research projects. Fresh ideas 
can also be tested almost instantly against real data so that they can be improved quantitatively.

\section{Calculations of Ionospheric Parameters}

For one of the active projects currently underway using GEDAS, we have begun to collect ground magnetometer data on a near real-time basis in an attempt to compute the worldwide distribution of ionospheric parameters, such as ionospheric electric fields and currents. Figure 1 outlines how this project operates. Beginning with ground-based observations, our interest lies, as indicated by the main vertical line, in estimating ionospheric electric fields and currents at high latitudes. We also obtain field-aligned currents which connect the high-latitude ionosphere and the outer magnetosphere. This is a typical example of the so-called inversion problems.

We intend to use real-time, or near real-time, ground magnetometer data eventually from 50-70 observatories, which are combined with data from more direct observations by satellites and radars. This joint effort of STEL, the NOAA National Geophysical Data Center (NGDC), the NOAA Space Environment Center, the National Center for Atmospheric Research, and the

University of Michigan, will use operationally updated versions of the KRM and AMIE programs (Kamide et al., 1981; Richmond and Kamide, 1988; Ridley et al., 1998) to compute the instantaneous, two-dimensional distribution of ionospheric electrodynamic parameters at high latitudes.

The uneven distribution of ground magnetometers on the earth's surface is one of the inevitable problems we are facing. The AMIE code, along with solar wind observations (Zwickl et al., 1998), is first used to calculate the overall distribution of the electric potential, which represents more or less a statistical pattern of the ionospheric potential, commensurate with the solar wind condition. For this process, an empirical model and ground magnetometer data only from a selected set of observatories are utilized. Once the global pattern is calculated, we use the result as the boundary condition to calculate more detailed structures of ionospheric parameters in a limited region on the basis of the KRM method (e.g., Sato et al., 1995).

In our practical scheme, three algorithms are currently being referred to: KRM, rt-AMIE, and local-KRM. The KRM algorithm was originally designed to calculate ionospheric parameters, such as electric fields and currents and field-aligned currents, as well as Joule heating, on the basis of ground magnetometer data. It first computes the magnetic potential that is a best fit to the distribution of ground magnetic perturbations and then estimates electric potential patterns in the ionosphere using an ionospheric conductance model. The real-time AMIE algorithm, rt-AMIE in short, is a simplified version of AMIE which is a technique of calculating similar ionospheric parameters from all available information, such as ground magnetometer data and satellite observations of field-aligned currents, and even 


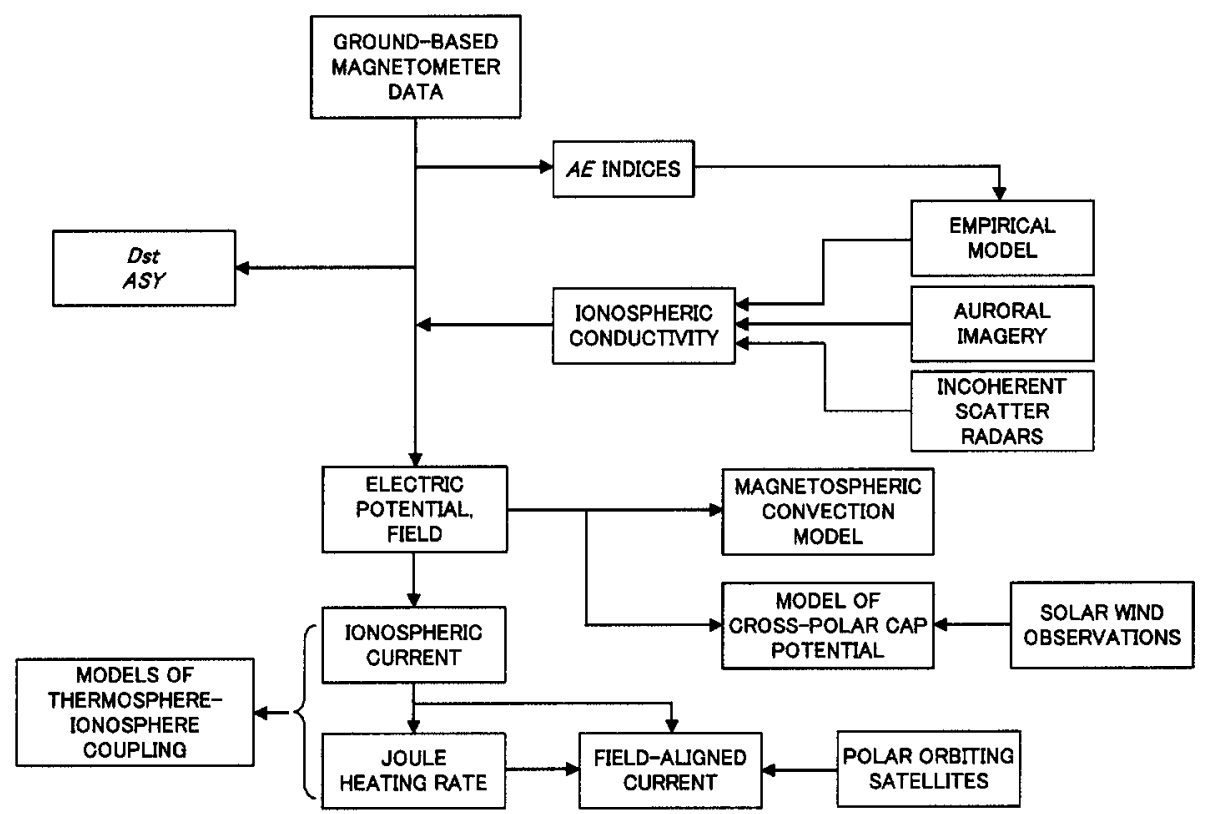

Figure 1. One of the GEDAS projects using the magnetogram-inversion technique, in which several ionospheric parameters are to be estimated on a real-time basis. The output will become important input for simulation/modeling studies at other institutions.

from empirical models of electrostatic potentials. The rt-AMIE algorithm that we are currently using depends on statistical models of the electric potential developed by Papitashvili et al. (1994) and Weimer (1995). These potential patterns in the models are given as a function of solar wind conditions, measured by the ACE spacecraft. The KRM and rt-AMIE algorithms have their own advantages and certain disadvantages. Relying on the strengths of each algorithm, a new algorithm, which is called the local-KRM algorithm, has been installed at GEDAS (Shirai et al., 2002). Local-KRM is in a sense an effective combination of KRM and rtAMIE. These two are used for separate estimates of ionospheric parameters in regions or local time sectors with good station coverage (by KRM) and poor coverage (by rt-AMIE). Care is taken to remove discontinuities, across the boundaries between these different regions.

At present, the number of stations providing real time, or near real-time, data is between $20-50$, depending on the availability of data. Figure 2 presents a schematic diagram showing data flow in our scheme. First, ground magnetometer data and solar wind data from ACE are assembled. These data are being used for running the rt-AMIE program at NOAA/NGDC. The output from rt-AMIE, along with the original magnetometer data, is forwarded to GEDAS and is used as the boundary condition for the local-KRM calculation.

The local-KRM output includes equivalent currents, electric potential patterns, ionospheric currents, and field-aligned currents. At present we are calculating these 


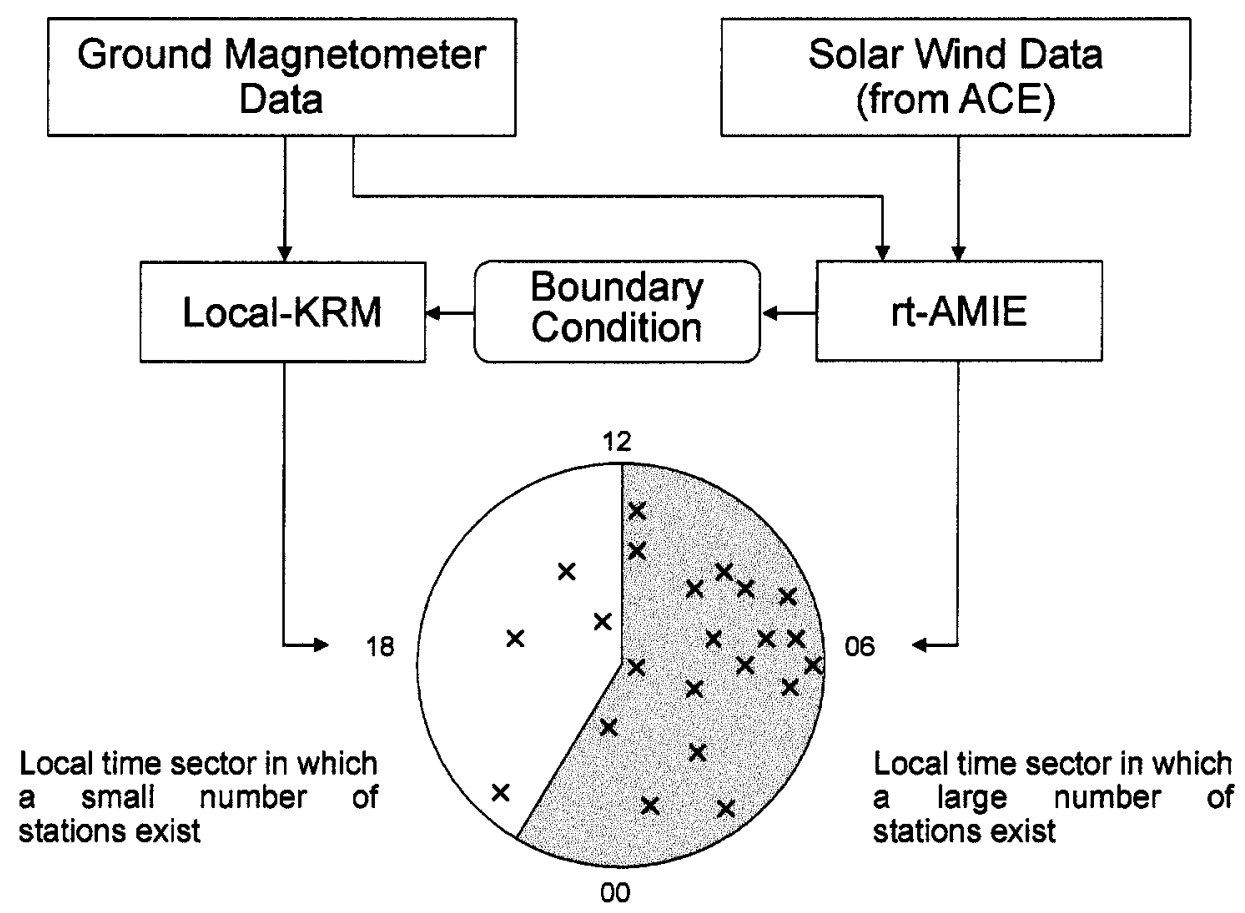

Figure 2. Schematic illustration showing data flow of the present project. Calculations of rt-AMIE are conducted at NOAA/NGDC whereas those of local-KRM are at STEL.

ionospheric parameters every 10 minutes with a grid size of 1 degree in latitude and $1 \mathrm{hr}$ in MLT, using the ionospheric conductivity model of Ahn et al. (1998): see http://gedas22.stelab.nagoya-u.ac.jp/index.html. The whole procedure, from ground-based observations to the local-KRM output, takes at present about 2030 minutes, depending on how quickly the data transport and actual calculations can be made.

Figures $3 \mathrm{a}$ and $\mathrm{b}$ present examples of the electric potential calculated for 1410 UT of July 17, 2002, just before the WISER workshop in Adelaide, from rt-AMIE (based on the Weimer (1995) model) and local-KRM, respectively. It is seen that the two patterns are quite similar to each other in that typical twin-vortex potential patterns can be identified in both potential distributions. The dusk-side pattern is nearly a duplication of that of rt-AMIE of the Weimer model. A potential minimum can be seen in 17-19 MLT hours at latitudes near $68^{\circ}$ in both distributions. One can notice that in the morning sector, where data from a number of stations were available, details of the potential patterns in the two plots differ from each other, including the MLT extension of the peak potentials. The difference in the total potential difference between the two methods is attributable to an underestimate of the maximum potential on the dawn side in the rt-AMIE calculation resulting from a statistical model in rt-AMIE. 


\section{ELECTRIC POTENTIAL}

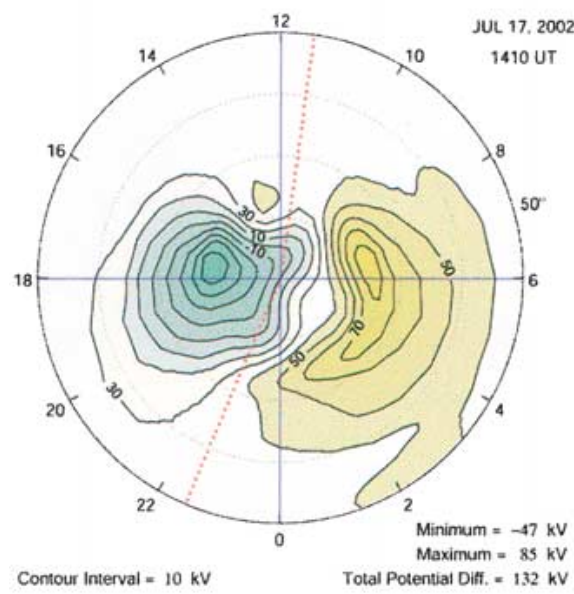

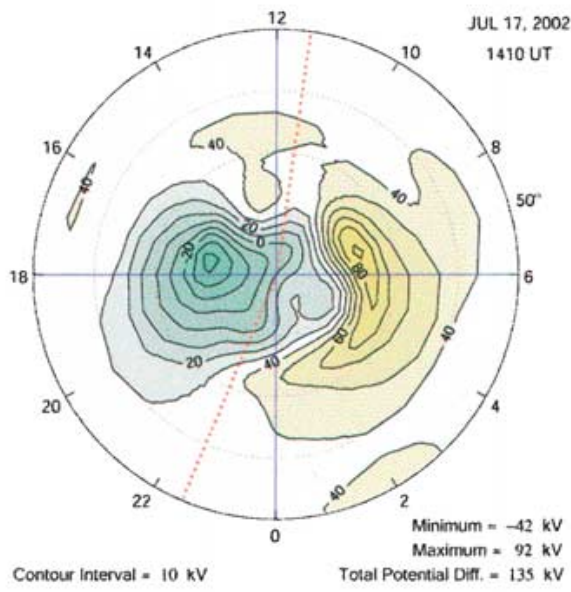

Figure 3. Example of real-time calculations of the electric potential: (a) global calculations made at NGDC on the basis of rt-AMIE; (b) local-KRM calculations made at STEL/GEDAS. Two MLT sectors are divided by a red dotted line. On the dawn side, data from a large number of magnetometers were available at this UT, whereas data from only a small number of magnetometers were available on the dusk side. The outer circle is for $50^{\circ}$ in geomagnetic latitude.

\section{IONOSPHERIC CURRENT VECTORS}

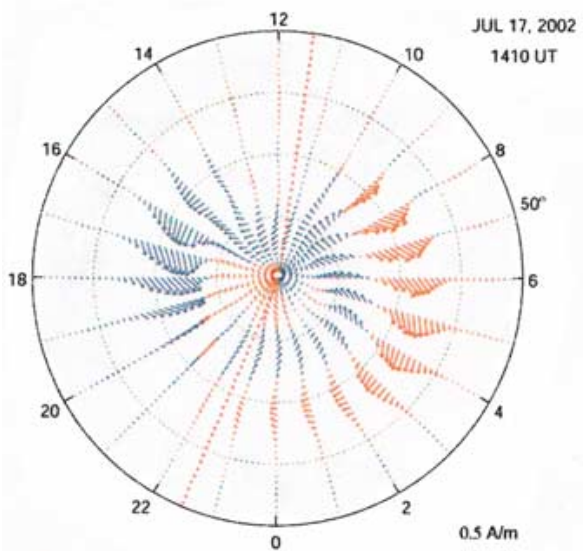

Blue: eastward
$0.5 \mathrm{Am}$

\section{FIELD-ALIGNED CURRENTS}

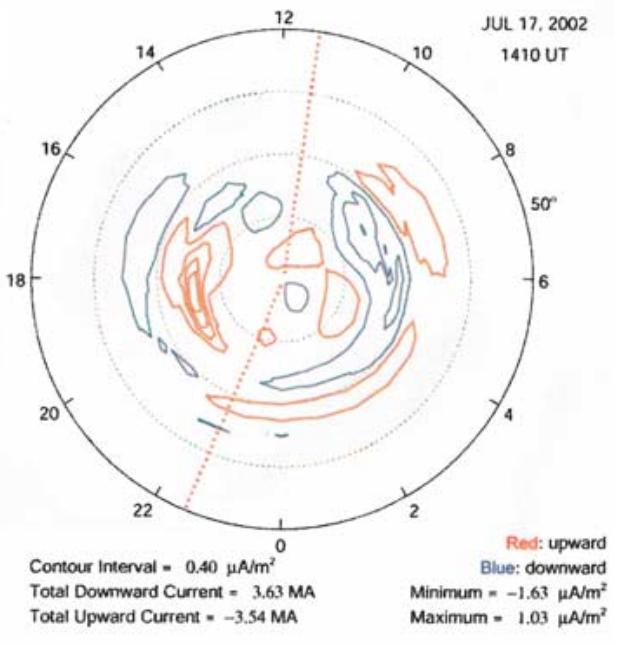

Figure 4. Local-KRM output, corresponding to the electric potential distribution shown in Figure 3:

(a) Ionospheric current vectors; (b) field-aligned currents. The outher circle is for $50^{\circ}$ in geomagnetic latitude. 
The distributions of ionospheric current vectors and field-aligned currents displayed in Figures 4a and 4b, respectively, show a pair of the eastward and westward auroral electrojets and the so-called Region 1/2 field-aligned currents. Since an intense substorm expansion was not in progress at this time, the overall distribution of the ionospheric currents and their divergence is not characterized by an intrusion of an intense westward electrojet into the midnight sector, such that the eastward and westward electrojets are rather 'nicely' separated. The corresponding fieldaligned currents, in which Region 1 current dominates, were also centered at dawn and dusk hours.

As Figure 1 indicates, the ionospheric conductivities can be normalized in the future using real-time observations by radars and global auroral distribution by polar-orbiting satellites. Once the ionospheric parameters are computed in this way, the output can be sent to institutions around the world where our output will become input for other modeling studies. For example, our electric field distribution can be mapped to the magnetosphere and is therefore useful for tracing particles in the magnetosphere: see Kamide et al. (2000) for some preliminary studies. Joule heating from our calculations can be used as input for calculating neutral winds in the thermosphere, which will modify the original electric field. There is no doubt that our output is also valuable for understanding the 'present' status of the auroral electrojets, which are critical to forecasting the strength of induced currents (Boteler et al., 1998; Pirjola et al., 2000).

\section{Related GEDAS Projects}

Other ongoing projects in the GEDAS framework include the following:

Solar Wind-Magnetosphere Coupling: An MHD simulation model of solar wind/magnetosphere interactions (Ogino et al., 1994) is in operation in real time using the solar wind data from ACE. The validity of the potential distribution calculated from this MHD model can be tested immediately by comparing with the AMIE/KRM calculations. Any inconsistency or disagreement between the results from the two approaches must be accounted for in terms of the assumptions employed in the modeling techniques. In the present case, the inconsistency or disagreement results primarily from the 'one way' MHD simulation in which no relevant ionospheric boundary condition is included. Relying on the realistic distribution of the ionospheric potential from the KRM/AMIE calculations, the simulations can be upgraded.

Prediction of Solar Wind Speed: To better understand the propagation of CMEs, corotating interaction regions, and other structures, observations of interplanetary scintillation (IPS) of natural radio sources are conducted on a routine basis at STEL (Kojima et al., 1998). Since IPS measurements are biased by line-of-sight integration, however, a computer assisted tomography technique is employed to obtain the 
longitudinal and latitudinal distributions of the speed and electron density fluctuations (Jackson et al., 1998). Solar wind predictions are being performed using this program, in which IPS data are transferred to the University of California at San Diego in near real-time. Predictions of the solar wind speed near the Earth for the following days are being derived from this tomography technique. The predicted solar wind speed is displayed on the world-wide-web (http://www.sec.noaa.gov/ace/ MAG_SWEPAM_24h.html) along with the ACE-observed solar wind speed.

\section{Outlook}

This paper has presented the recent progress we have made in our joint GEDAS program, with a special emphasis on the near real-time specification of ionospheric electrodynamics. The scheme we have developed can provide the scientific community with two-dimensional mapping of electric fields and currents in the ionosphere, as shown in Figures 3 and 4. Our final goal is to predict accurately space weather events when all details are coded properly in the computer system and when the 'present' condition of the sun is given. There should be no doubt that in order to achieve this degree of accuracy, a super computer of extremely high speed and capacity would be necessary.

Toward this goal, the project we have described in this paper is quite promising in, at least, two respects. First, GEDAS provides the scientific community with specifications of the geospace environment well beyond what are available from the popular geomagnetic activity indices. In addition, since the data products the GEDAS programs provide are based on real-time recordings of magnetic perturbations from a number of stations, the output should be more realistic than average potential patterns using a large number of 'point' measurements. By now it is well understood that the sun does not decide everything that will occur in the near-earth environment, and that the solar wind gives only the boundary condition for the magnetosphere in which various nonlinear plasma processes take place.

Second, it is important to note that recent space weather modeling efforts including global MHD and other modular models have also been improved, beginning to contribute to understanding the effects of solar wind disturbances on the magnetosphere and ionosphere (e.g., Raeder et al., 1998; Papadoupoulos et al., 1999; Gombosi et al., 2000; Tanaka, 2000). It is important to integrate these simulation models and real-time observations to increase our fidelity of space weather specifications and predictions. We are confident that computer systems, such as GEDAS, will contribute considerably to this integration.

Finally, two areas are noted which are of crucial importance for the success of space weather studies. They are the need for effective combinations (or interplays) of: (1) Observations and Modeling, and (2) Nowcasting and Forecasting. These will hopefully be accomplished within the GEDAS concept. 
(1) Observations and Modeling: Spacecraft observations alone are inadequate to cover every plasma region in the solar-terrestrial system. Under appropriate initial/boundary conditions using real-time observations in the upstream solar wind, a numerical solution can be achieved, which would then be used to predict the next magnetospheric and ionospheric event, the validity of which would be readily checked against real-time data.

(2) Nowcasting and Forecasting: As demonstrated in the present paper, it is now possible to nowcast the global distribution of the electric potential in the ionosphere primarily from ground-based magnetometer observations. The time history of this potential distribution, which is very similar to the air pressure weather maps, can be extrapolated to the next moment using some efficient statistical methods. We must realize that the solar wind alone do not determine every process in the magnetosphere and ionosphere, but the history of the inner magnetosphere and the polar ionosphere determines where and how a substorm of what magnitude will take place.

\section{Acknowledgements}

We are grateful to Dr. H. Shirai who developed an early scheme of the local-KRM program, and Drs. V. O. Papitashvili and D. R. Weimer who kindly provided their models for our project. We also thank Drs. B.-H. Ahn, H. W. Kroehl, and A. S. Sharma for their illuminating discussions during the preparation of this manuscript.

\section{References}

Ahn, B. -H., Richmond, A. D., Kamide, Y., Kroehl, H. W., Emery, B. A., de la Beaujardire, O. and Akasofu, S. -I.: 1998, 'An Ionospheric Conductance Model Based on Ground Magnetic Disturbance Data', J. Geophys. Res. 103, 14,769.

Boteler, D. .H., Pirjola, R. J. and Nevanlinna, H.: 1998, 'The Effects of Geomagnetic Disturbances on Electrical Systems at the Earth's Surface', Adv. Space Res. 22, 17.

Gombosi, T. I., De Zeeuw, D. L., Groth, C. P. T. and Powell, K. G.: 2000, 'Magnetospheric Configuration for Parker-spiral IMF Conditions: Results of a 3D AMR MHD Simulation', Adv. Space Res. 26, 139.

Jackson, B. V., Jick, P. L., Kojima, M. and Yokobe, A.: 1998, 'Heliospheric Tomography using Interplanetary Scintillation Observation, 1. Combined Nagoya and Cambridge data', J. Geophys. Res. 103, 12,049.

Kamide, Y., Richmond, A. D. and Matsushita, S.: 1981, 'Estimation of Ionospheric Electric Fields, Ionospheric Currents, and Field-aligned Currents from Ground Magnetic Records', J. Geophys. Res. 86, 801.

Kamide, Y., Shue, J. -H., Hausman, B. A. and Freeman, J. W.: 2000, 'Toward Real-time Mapping of Ionospheric Electric Fields and Currents', Adv. Space Res. 26, 213.

Kojima, M., Tokumaru, M., Watanabe, H., Yokobe, A., Asai, K., Jackson, B. V. and Hick, P. L.: 1998, 'Heliospheric Tomography using Interplanetary Scintillation Observation, 2. Latitude and Heliospheric Dependence of Solar Wind Structure at 0.1-1 AU', J. Geophys. Res. 103, 1981. 
Ogino, T., Walker, R. J. and Ashour-Abdalla, M.: 1994, 'A Global Magnetohydrodynamic Simulation of the Response of the Magnetosphere to a Northward Turning of Interplanetary Magnetic Field', J. Geophys. Res. 99, 11,027.

Papitashvili, V. O., Belov, B. A., Faermark, D. S., Feldstein, Y. I., Golyshev, S. A., Gromova, L. I. and Levitin, A. E.: 1994, 'Electric Potential Patterns in the Northern and Southern Polar Regions Parameterized by the Interplanetary Magnetic Field', J. Geophys. Res. 99, 13,251.

Papadoupoulos, K., Goodrich, C. C., Wiltberger, M., Lopez, R. E. and Lyon, J. G.: 1999, 'The Physics of Substorms as Revealed by the ISTP', Phys. Chem. Earth 24, 189.

Pirjola, R., Viljanen, A., Pulkkinen, A. and Amm, O.: 2000, 'Space Weather Risk in Power Transmission Systems', Phys. Chem. Earth 25, 333.

Raeder, J., Berchem, J. and Ashour-Abdalla, M.: 1998, 'The Geospace Environment Modeling Grand Challenge: Results from a Global Geospace Circulation Model', J. Geophys. Res. 103, 14,787.

Richmond, A. D. and Kamide, Y.: 1988, 'Mapping of Electrodynamic Features of the High-latitude Ionosphere from Localized Observations: Technique', J. Geophys. Res. 93, 5741.

Ridley, A. J., Moretto, T., Ernstroem, P. and Clauer, C. R.: 1998, 'Global Analysis of Three Traveling Vortex Events during the November 1993 Storm using the Assimilative Mapping of Ionospheric Electrodynamic Technique', J. Geophys. Res. 103, 26,349.

Sato, M., Kamide, Y., Richmond, A. D., Brekke, A. and Nozawa, S.: 1995, 'Regional Estimation of Electric Fields and Currents in the Polar Ionosphere', Geophys. Res. Lett. 22, 283.

Shirai, H., Kamide, Y., Kihn, E. H., Hausman, B., Shinohara, M., Nakata, H., Isowa, M., Takada, T. K., Watanabe, Y. and Masuda, S.: 2002, 'Near Real-time Calculation of Ionospheric Electric Fields and Currents using GEDAS', Proc. of COSPAR Coll. (in press).

Tanaka, T.: 2000, 'The State Transition Model of the Substorm Onset', J. Geophys. Res. 105, 21,081.

Weimer, D. R.: 1995, 'Models of High-latitude Electric Potentials Derived with a Least Error Fit of Spherical Harmonic Coefficients', J. Geophys. Res. 100, 19,595.

Zwickl, R. D., Doggett, K., Sahm, S., Barrett, B., Grubb, R., Detman, B. R., Raben, V. J., Smith, C. A., Riley, P., Gold, R. E., Mewaldt, R. A. and Maruyama, T.: 1998, 'The NOAA Real-Time Solar-Wind (RTSW) system using ACE data', Space Sci. Rev. 86, 633. 